Research Journal of Applied Sciences 13 (10): 594-602, 2018

ISSN: $1815-932 \mathrm{X}$

(C) Medwell Journals, 2018

\title{
Reconfigurable Hardware Features of Modular Robotic System MecaBot
}

\author{
Ricardo Andres Castillo Estepa, David Jesus Gomez Ceballos and German Andres Vargas Torres \\ DaVinci Research Group, Universidad Militar Nueva Granada, Bogota, Colombia
}

\begin{abstract}
The MecaBot is a robotic, modular and reconfigurable system capable of adding new modules to form various configurations depending on the type of terrain it traverses and the different types of obstacles it has to overcome. This system was designed and implemented in Nueva Granada Military University as a research and education platform. The aim of this reserach is to present the different configurations and possible connection modes that can be achieved according to the desired topology of the module it shows the evolution of the system from the first version to the last making comparisons between versions 3 and 4 mainly, showing the capabilities of each in the configurations for which they were implemented and a description of the components and their functions within the system.
\end{abstract}

Key words: Modular robotics, module, self-reconfiguration, simulation, platform, implemented

\section{INTRODUCTION}

The main component in modular robotics are the robotic agents that integrate the system which in hardware terms can be identical (homogeneous) or have variations that allow parts of the system to specialize in performing specific functions, since, it is desirable for agents working in unknown environments with obstacles to have features which allow them to overcome these hazards as a cooperating group.

For robotic agents, manufacture methods bear equal significance to the robot's design given the relation between said methods and production costs. Agile fabrication of large quantities of agents at low cost is desirable for modular robotics.

Prototypes of modular robotic system MecaBot-3 were produced by rapid prototyping or $3 \mathrm{D}$ printing by Fused Deposition Modeling (FDM) using ABS plastic which is a strong yet lightweight material that now a days can easily be found commercially on the market. Such qualities are paramount in structures subject to directional mechanical strain.

The steady growth of 3D printing, mainly dedicated personal 3D printers, points towards an increase in accessibility and a reduction of entry costs for researchers and roboticists is a key advantage for construction of complex modular robots and spare parts.

\section{MATERIALS AND METHODS}

Modular robotics: The concept of modular robotics comes from the proposal of John Von Newmann called
"Theory of self-reproducing cellular automata" which sought to buildsystems using homogeneous components. From these theories, engineer Mark Yim at Stanford University designed reconfigurable modular robots based on a chain-type architecture. His 1994 doctoral thesis ("Locomotion with a unit-modular reconfigurable robot") proposed the polypod (Yim, 1994). Despite previous studies in modular robotics, polypod was the first robot of its kind to use the concept of reconfiguring systems.

Similar systems like CEBOT or Fracta emerged shortly thereafter. CEBOT had different types of modules designed for specific tasks such as transportation, grip, rotation modules and arms among others which allowed it to perform various tasks (Murata and Kurokawa, 2007).

The aim of reconfigurable modular robotics are systems composed of several elements called modules which can couple/decouple in multiple ways to acquire various skills not possible for an individual robot (Yim et al., 2002).

Currently there are several modular robots built using open-source 3D printing such as PMR (The Printable Modular Robot) (Anonymous 2016a, b) and cube revolutions (chain architecture) developed by John Gonzalez Gomez (Matellan and Canas, 2006) and shown in Fig. 1. The robot consists of 8 Y1-type modules connected in a serial kinematic chain with pitch-pitch direction (perpendicular to the plane of rotation movement). The position of each of the servomotors is determined by a microcontroller which receives instructions from an interface developed in a computer connected via. RS-232.

Corresponding Author: Ricardo Andres Castillo Estepa, DaVinci Research Group, Universidad Militar Nueva Granada, Bogota, Colombia 


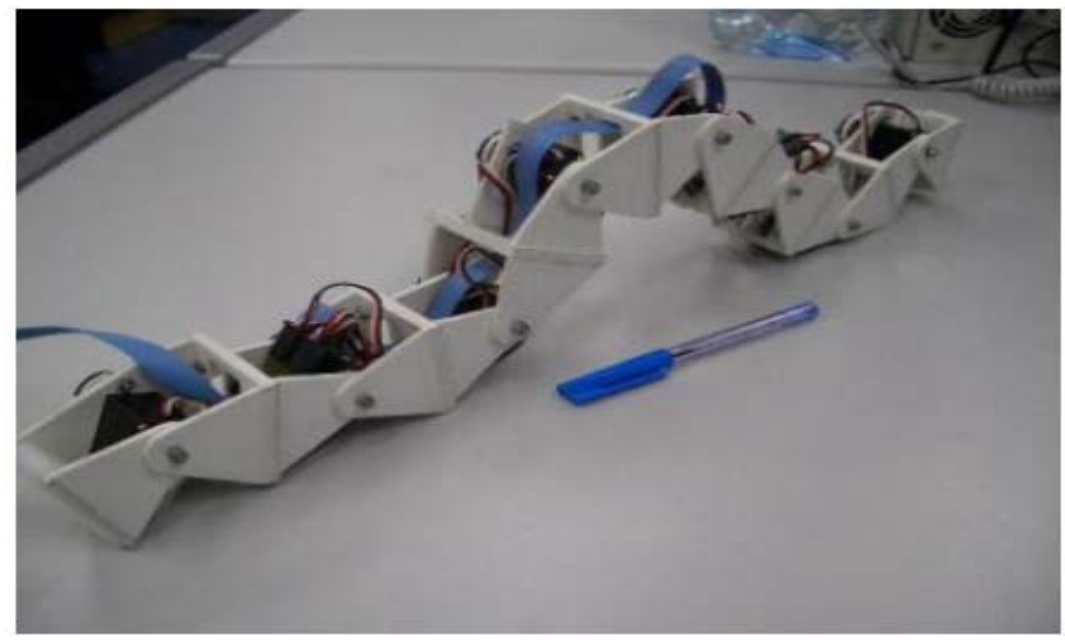

Fig. 1: Cube revolution robot in chain configuration. This robot is legless, modular and manually reconfigurable (Matellan and Canas, 2006)

(a)

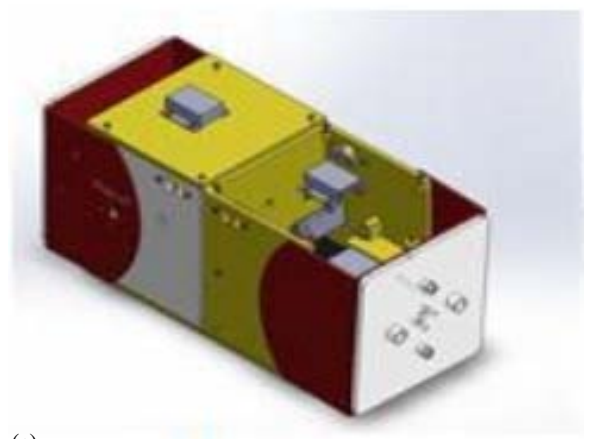

(c)

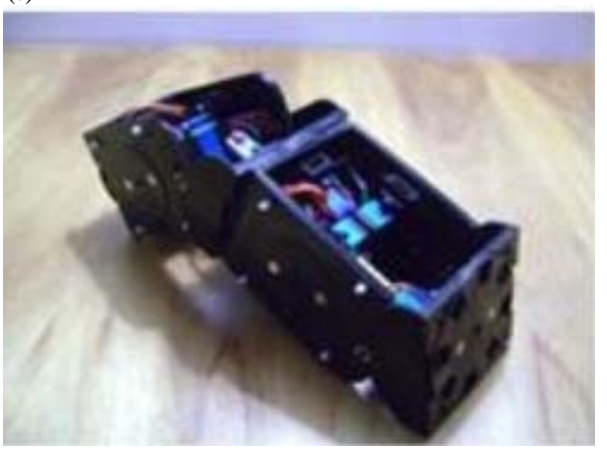

(b)

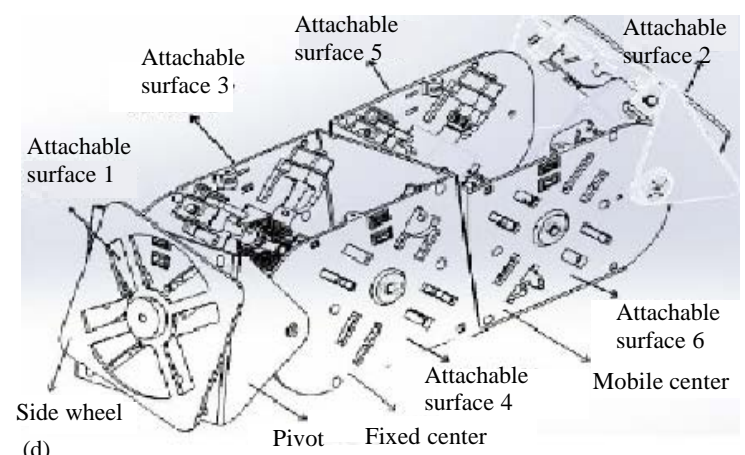

(d)

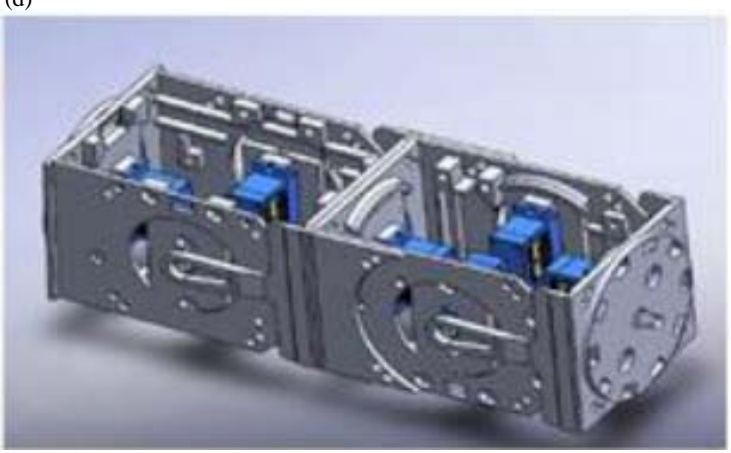

Fig. 2: Evolution of system modules MecaBot (Montana and Hurtado, 2013); a) MecaBot 1; b) MecaBot 2; c) MecBot 3 and d) MecaBot 4

Evolution of MecaBot: In order to define design characteristics and prepare for potential problems, the first step in robot design is an analysis of the tasks it is desired to perform. However, for modular robots this is not a concern since one of the main characteristics of these systems is the inherent capacity to adapt to the environment. Authors state that modular robotics has three main features: robustness, versatility and a low cost (Yim et al., 2002). To date, four versions of the system (as shown in Fig. 2) each with improvements over the previous one have been designed.

Robot shape, proportions and degrees of freedom are similar in the last two iterations, yet dimensions, coupling system and electronics were upgraded in the current 
(a)

(b)

(c)

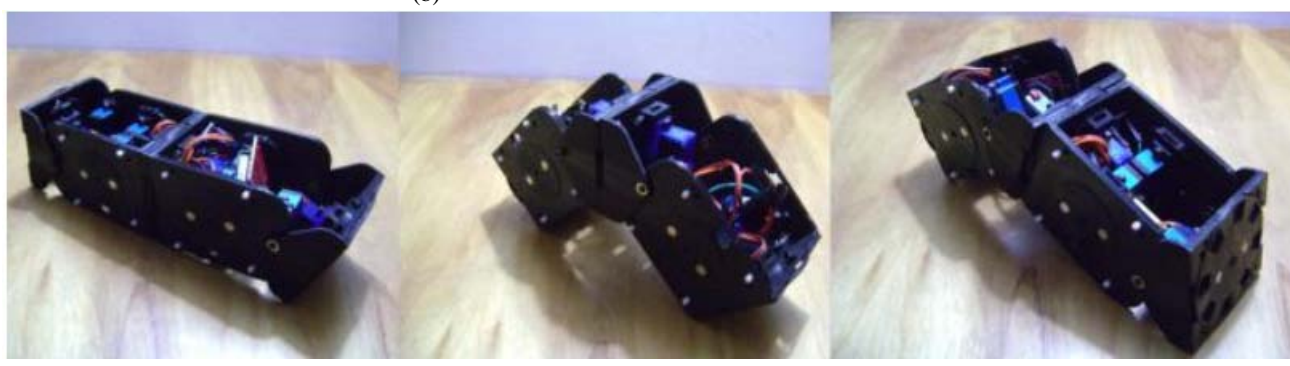

Fig. 3: Sub-module adjustments for MecaBot-3; a) Sub-configuration 1; b) Sub-configuration 2 and c) Sub-configuration 3

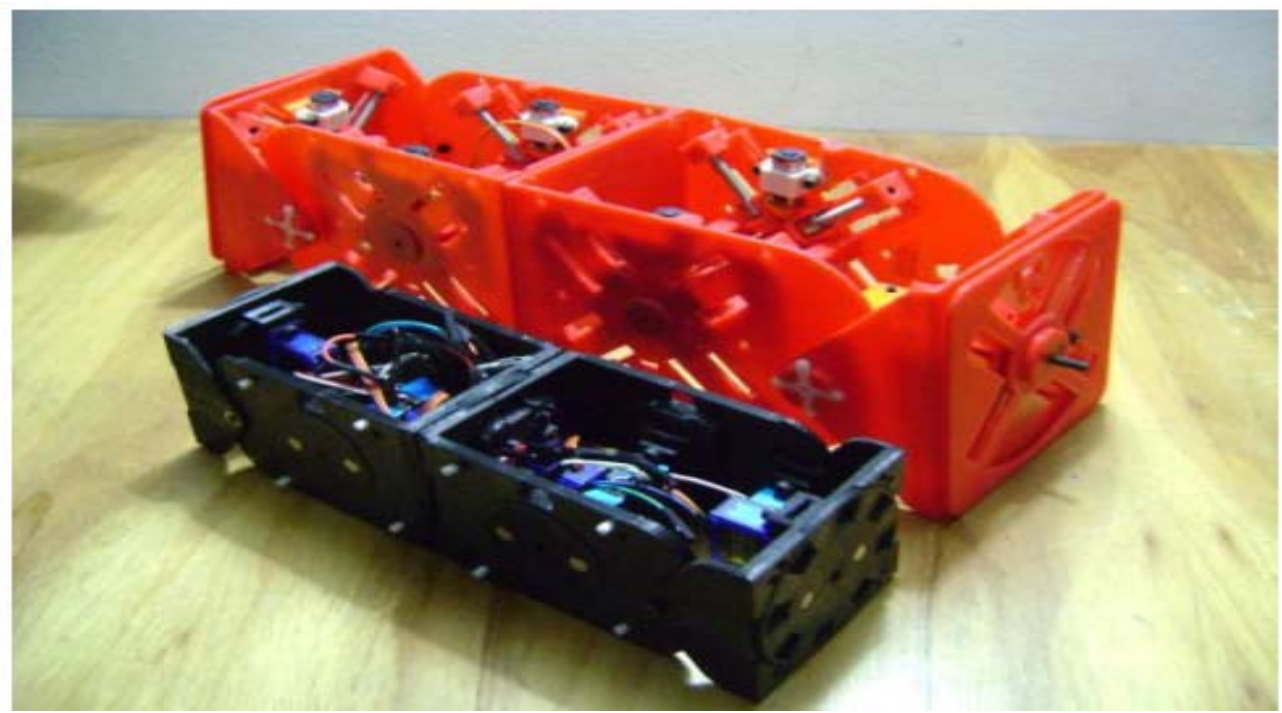

Fig. 4: Comparison between MecaBot-2 and 3

version. Features such as sub-modular separation capabilities, greater battery life and total weight reduction enhance its performance as a modular system.

An individual agent consists of two semi-modules, each with its own electronics, power supply and communication system. Each semi-module can not be considered an agent, due to the inability of movement (and by extension, operation) in uneven ground on its own. In consequence, it is necessary to couple two semi-modules to form a robotic agent that is totally independent and functional. The advantage of having sub-modular separation capabilities allows an agent adjustment of its physical footprint. Figure 3 presents three different adjustments, providing flexibility in construction of complex multi-module architectures.

Each semi-module has three Degrees of Freedom (DOF), yet, a complete module has five DOF no matter the arrangement distributed as follows: two side wheels, two in the pivots and one in the center which allows semi-modules to rotate relative to one another. All these DOF are driven by micro-servos; Use of these actuators allows for a much smaller robot than its predecessor: $6 \times 6 \times 10 \mathrm{~cm}$ for MecaBot-3 versus $10 \times 10 \times 15 \mathrm{~cm}$ for MecaBot-2. Figure 4 provides size comparison between MecaBot-2 and MecaBot-3, the size of the MecaBot-4 is equal to the size of the third version.

This size reduction also means a decrease in weight: each complete semi-module weighs approximately $125 \mathrm{~g}$ versus $375 \mathrm{~g}$ for the previous version. Lower weight is beneficial for reduced energy consumption and actuator wear.

Location of robot electronics is controlled by adjusting internal plates with screws. Since, MecaBot- 3 is a prototype, there is still not a single PCB board that integrates all system electronics. Figure 5 presents the robot housing's design which accommodates all electronics and Fig. 6 illustrates the electrical connections diagram of a semi-module. 


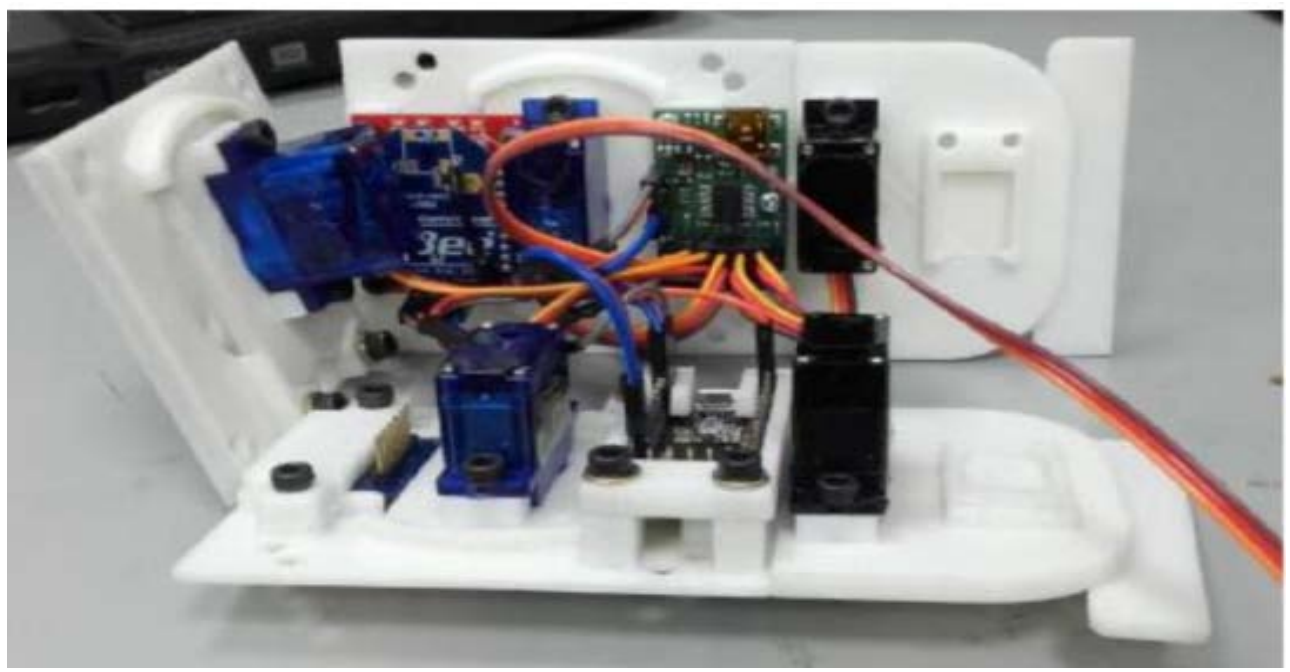

Fig. 5: Electronics on the semi-module's housing

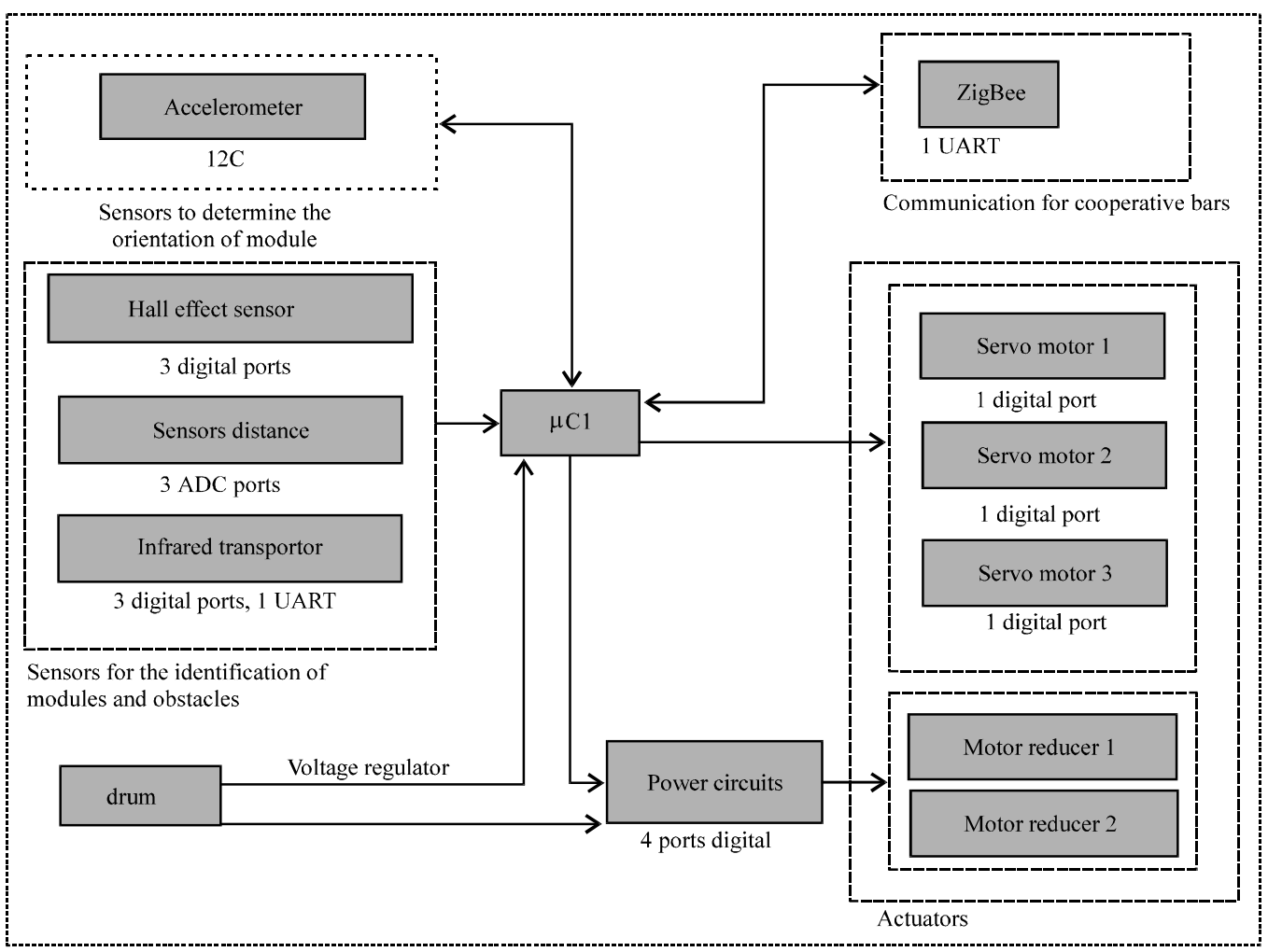

Fig. 6: Electrical connections diagram for a semi-module

\section{RESULTS AND DISCUSSION}

Docking system: When two modules are located at $1 \mathrm{~cm}$ from one another, the coupling mechanism works by rotating disks in the matching faces of both modules. These discs are two neodymium magnets positioned with reverse polarities as shown in Fig. 7.
Coupling discs on the module's faces may have two positions: attraction and repulsion. When coupling discs are mismatched, actuators in each module rotate the disks by $90^{\circ}$; The sum $\left(180^{\circ}\right)$ faces the magnets in the same polarity (repulsion) or opposite polarities (attraction).

It is not necessary to have perfect alignment between modules before coupling; guiding the magnets in the 


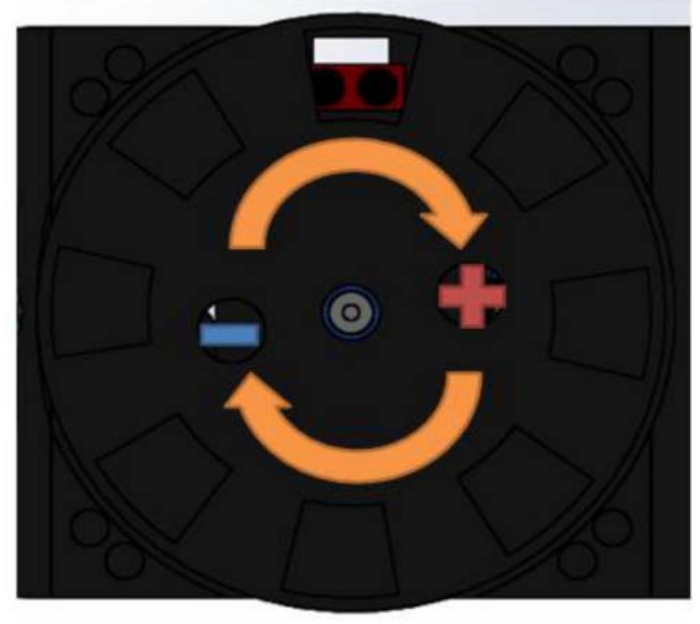

Fig. 7: Magnetic oupling system in MecaBot-3 and 4

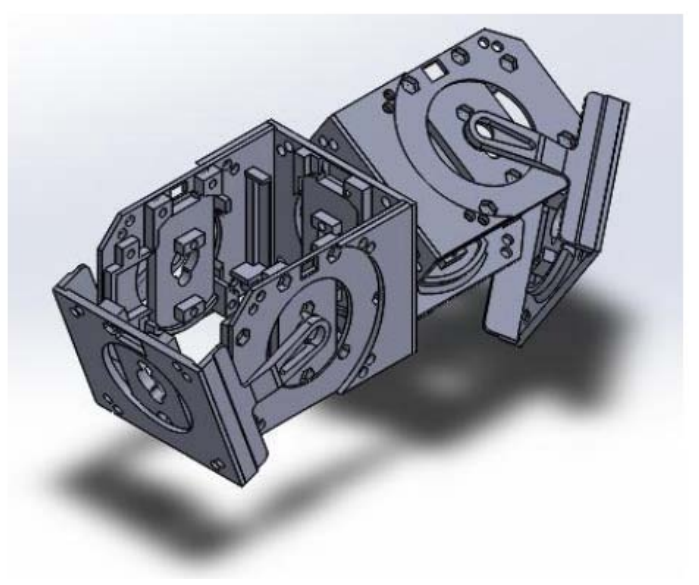

Fig. 8: MecaBot-4 face-face attachment

required position is sufficient. This is an advantage because the modules will work in uneven ground where precise alignment is difficult to achieve due to unpredictable environments.

Possible coupling configurations: Possible configurations depend largely on the individual structure of each semi-module, the degrees of freedom and the coupling method. Each semi-module has four mating surfaces, one on each side. All these surfaces are compatible with each other, thus, allowing different system configurations for operation in real environment.

With the current topology, the agents can form three coupling modes. Furthermore, the pivot can rotate $180^{\circ}$, providing many alternatives when making configurations, the first of which is shown in Fig. 8. This connection is

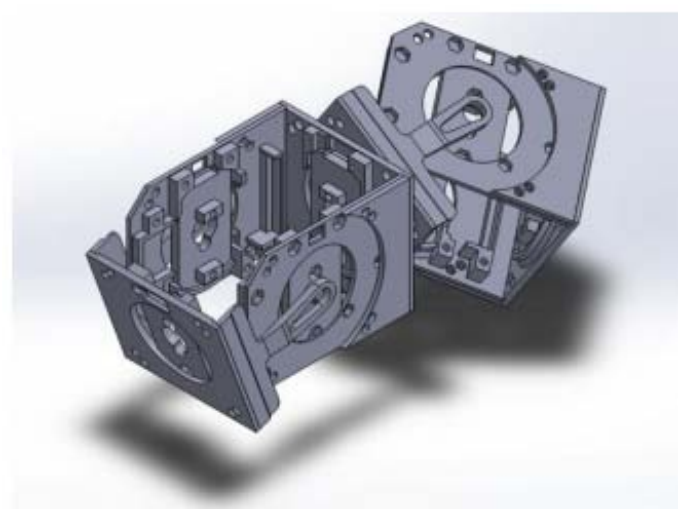

Fig. 9: MecaBot-4 face-pivot attachment

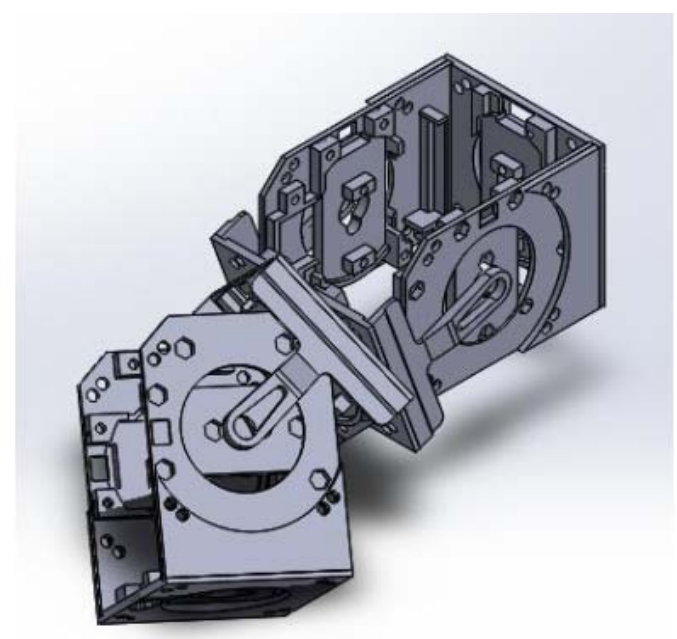

Fig. 10: MecaBot-4 pivot-pivot attachment

made with the union of two faces which can rotate in order to clear the pivot for further couplings between semi-modules or agents.

Figure 9 presents the second coupling mode which is formed by mating one semi-module's face with the other semi-module's pivot. In Fig. 10, the last coupling mode is shown: a pivot-pivot union. This unifies $3^{\circ}$ of freedom in a single point forming a joint akin to a spherical or wrist-type joint.

Using these coupling modes a variety of configurations with different characteristics can be formed, usable in different types of terrain to overcome various types of obstacles.

Figure 11 shows a hexapod-type robot built by combining face-face and face-pivot couplings. This provides the robot an additional degree of freedom in its center (waist) for more natural motions when traversing uneven terrain. 


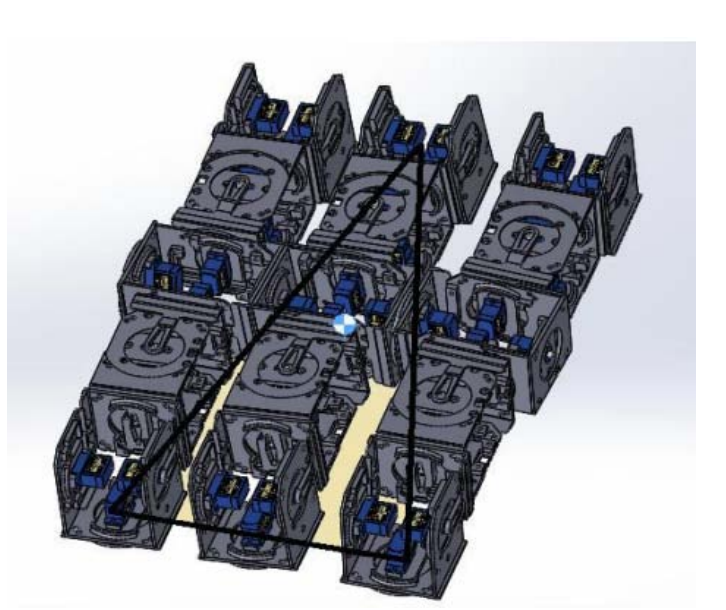

Fig. 11: MecaBot-4 hexapod configuration

Communication system: In a modular robotic system communication is essential to ensure coordination of all actors involved. Wired connections (done by physically connecting circuits using a cable capable of transmitting signals) are difficult to interrupt and are immune to noise interference depending on the cable's coating material (Krupke et al., 2015). However, they are limited by short range and require direct physical connections between agents.

Wireless connections do not require a wired medium to transmit the signal, so, agents can move independently, in the case of the modular robotic processes auto configuration become simpler. Amongst its disadvantages one can list energy consumption (High in comparison to wired connections) and susceptibility to interference and signal degradation due to other agents or obstacles present in the environment (Krupke et al., 2015).

Wireless communication is chosed for MecaBot-3 given the need for freedom of movement. Since, the robotic agents have sensors to interact with the environment, the complete system can be viewed as a network of wireless sensors. "Wireless sensor networks are based on small devices (nodes) that are able to get information from the environment, process it locally and send it wirelessly to a central node coordinator" (Anonymous, 2016a, b). Therefore, each semi-module acts as a node and the central node can be a computer or some agent named as a system leader.

For the semi-modules to function as nodes they must have the following elements: a microcontroller that processes data acquired from sensors and relays said data to a transceiver, a sensor responsible for generating electrical signals according to changes in the environment, a battery as a portable power source and a transceiver that sends and receives data from other

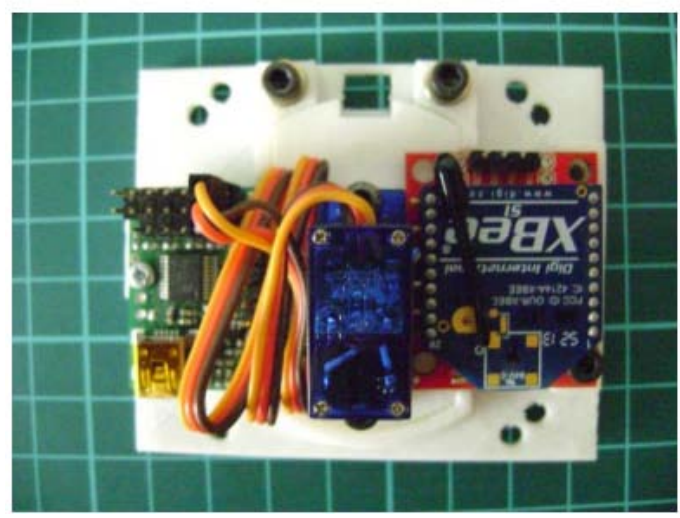

Fig. 12: MecaBot-3 wireless communications system

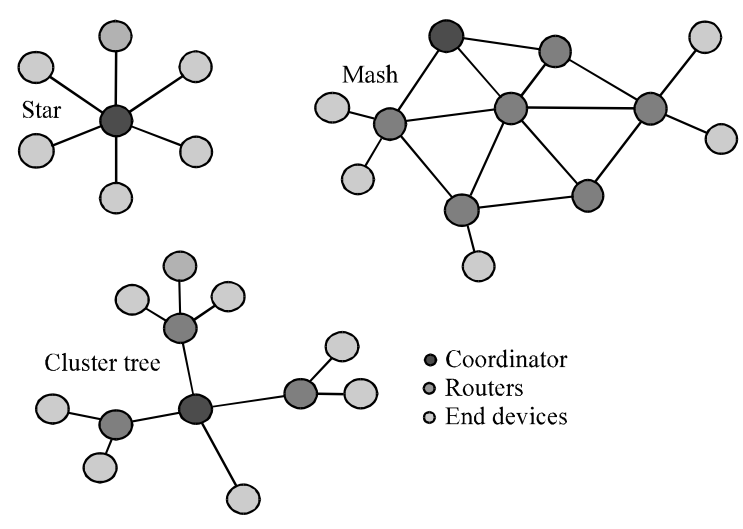

Fig. 13: Wireless network topologies

nodes. Figure 12 presents all communication components in a semi-module; XBee was the wireless module of choice.

The choice of topology for the wireless network depends on the system's control hierarchy: a star topology is best for centralized systems (control from a computer) whilst a mesh topology is better suited for systems with autonomous unsupervised agents. Both topologies are shown in Fig. 13 as a and b, respectively.

Actuators: The following Fig. 14 shows the degrees of freedom of a semi-module MecaBot-3. In order to implement the movement of the modules, servo motors were used. MG90S reference micro-servomotors, Fig. 15 were used in the main articulation (3DOF) which have metal pinion and can rotate approximately $180^{\circ}$.

\section{Specifications MG90S:}

- Weight: 13.4 (g)

- Torque: $2.3(\mathrm{kgf} \bullet \mathrm{cm})(6 \mathrm{~V})$

- Voltage: $4.8-6.0(\mathrm{~V})$ 
For central and face articulation (1DOF) SG90 micro-servomotors (Fig. 16) were used which have plastic pinion and smaller torque than those used in the main joint.

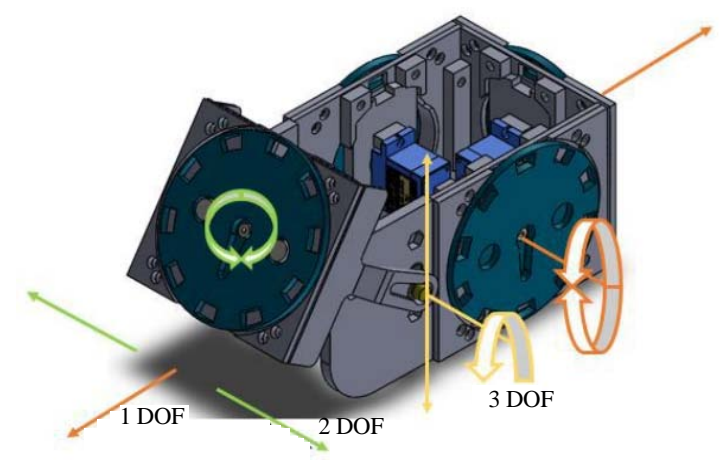

Fig. 14: Degrees of freedom MecaBot 3.0

\section{Specifications SG90:}

- Weight: $9(\mathrm{~g})$

- Torque: $1.8(\mathrm{kgf} \bullet \mathrm{cm})$

- Operation speed: $0.1 \mathrm{sec} / 60^{\circ}$

- Voltage: $4.8 \mathrm{~V}-5.0(\mathrm{~V})$

In the fourth version of the system were used higher torque motors due to the requirements of the configurations, the characteristics of the actuators used are as follows:

- Weight: $15.8(\mathrm{~g})$

- Torque: $3.9(\mathrm{kgf} / \mathrm{cm})$

- Voltaje: 4.8-6.0(V)

This actuator has a similar size to the one used previously but has a greater torque of greater energy consumption (Fig. 17).
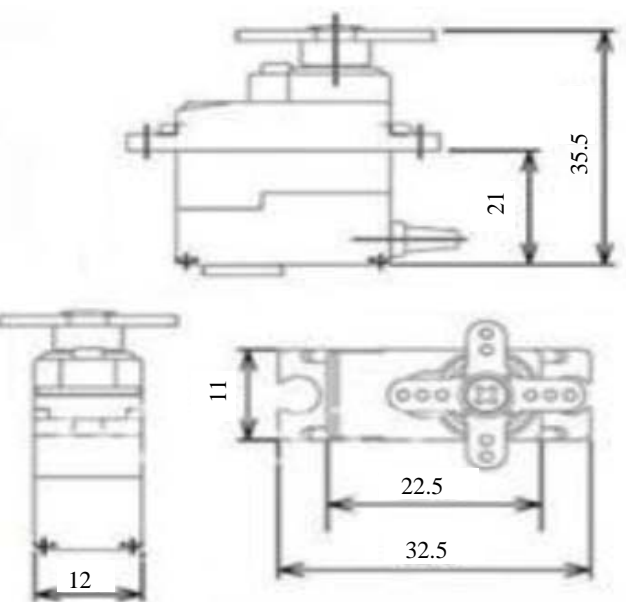

Fig. 15: Micro-servomotor MG90S
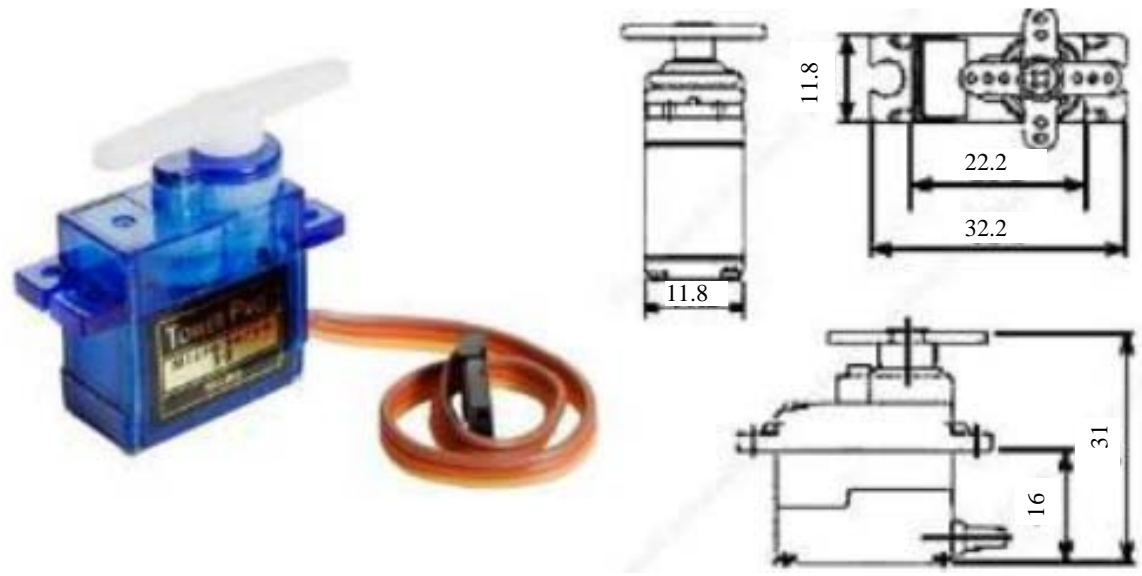

Fig. 16: Micro-servomotor SG90 


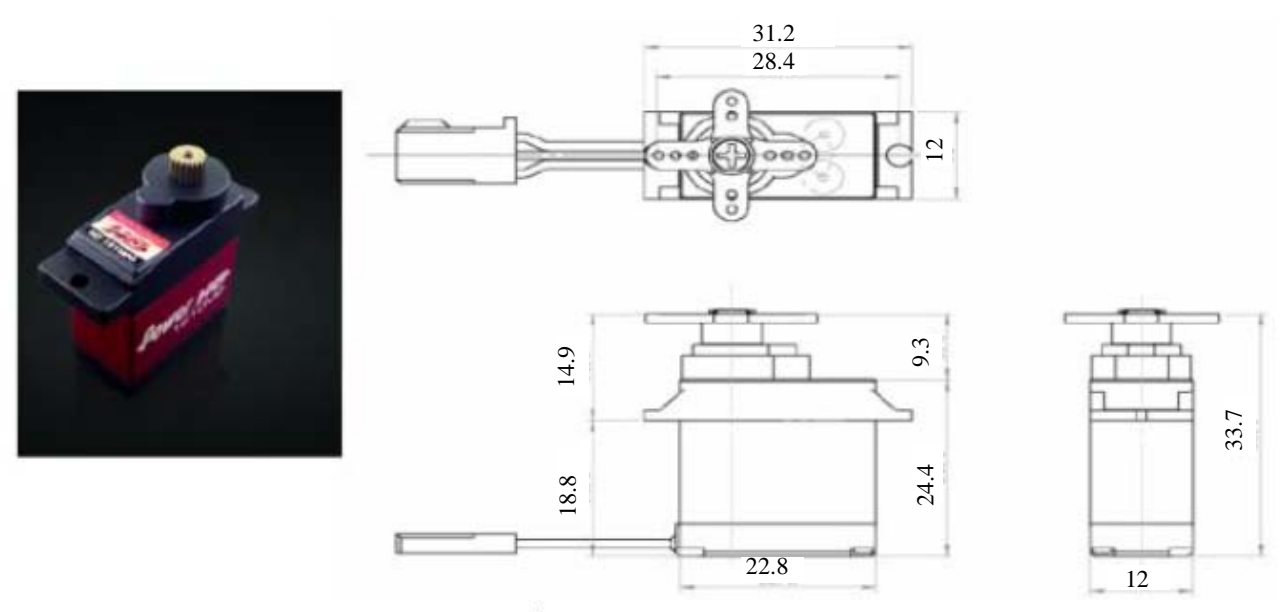

Fig. 17: Servomotor

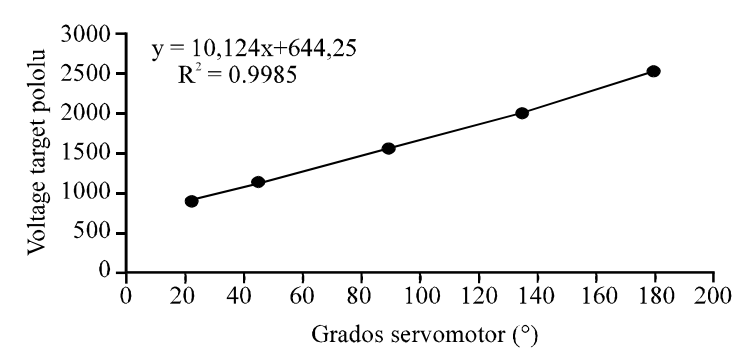

Fig. 18: Servomotor characterization

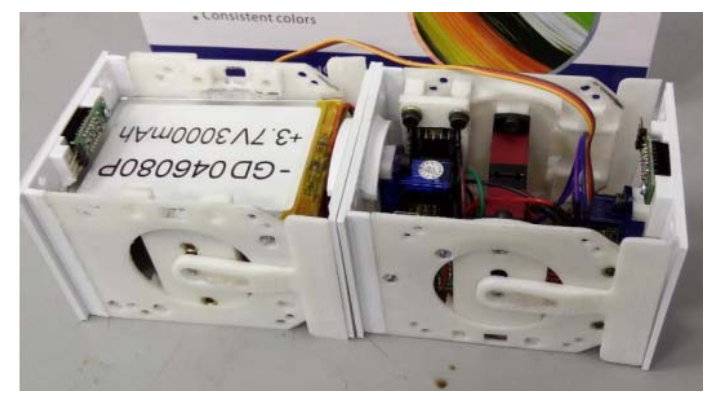

Fig. 19: Module MecaBot 4

different manufacturers. The equations of this pair of motors that make up each half-module are loaded into the command program.

MecaBot -4 and possible configurations: The MecaBot- 4 is an improvement of the system that was realized, so that, it was possible the implementation of the configurations wheel and hexapod, making modifications in the size and actuators.

For the redesign of the MecaBot-4 Fig. 19 the necessary conditions for the formation of the wheel and hexapod configurations were taken into account in these
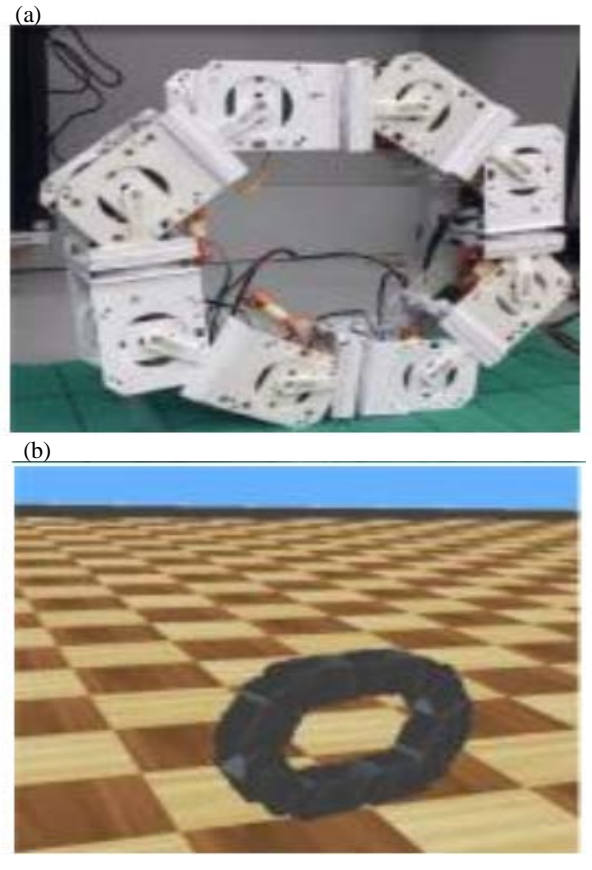

Fig. 20: Mechanical wheel configurationMecaBot 4

the torque in the joints must be recalculated, since, they are conformed with more modules which increases the total weight.

The MecaBot- 4 has an approximate weight of $200 \mathrm{~g}$ as well as the MecaBot-3 but unlike this one has a length of $19 \mathrm{~cm}$ which helps to decrease the torque in the joints, additionally has micro servos with a torque of $8 \mathrm{~kg} . \mathrm{cm}$, this reduction size and increased strength in the joints make possible the formation of wheel and hexapod configurations.

For the wheel configuration, Fig. 20 it is necessary that a module must be able to lift 1.5 robot from the 


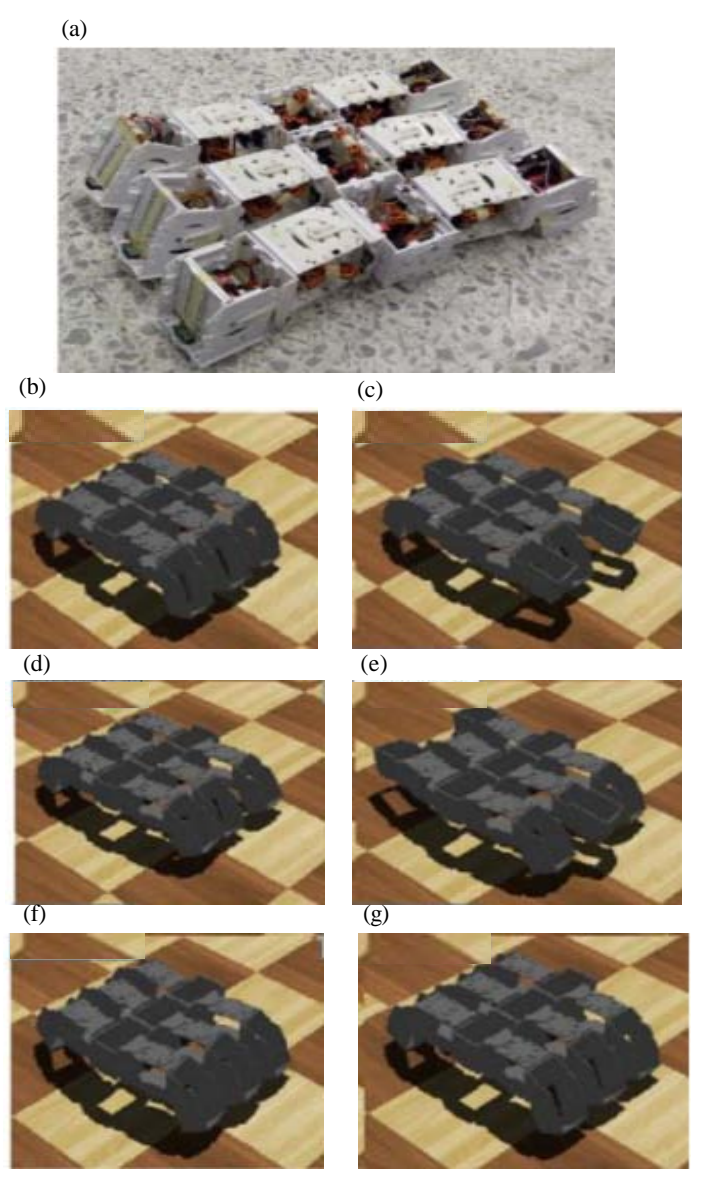

Fig. 21: a) Configuration hexapod MecaBot-4, built model and running simulation; b) Inicio; c) Adelante 1; d) Adelante 2; e) Adelante 3; f) Adelante 4 and g) Adelante 5

system because it is made up of 4 modules there will always be one at the bottom that must support the remaining three at two points of contact its extremes, taking into account that the weight of a MecaBot-4 module is $200 \mathrm{~g}$ and has a length of $16 \mathrm{~cm}$ requires a torque of $0.55 \mathrm{~N} / \mathrm{m}$ which is approximately $5.5 \mathrm{~kg} / \mathrm{cm}$. With these conditions, metallic servomotors of $4 \mathrm{~kg} . \mathrm{cm}$ were selected as they meet the requirement of size and torque when working together generating $8 \mathrm{~kg} / \mathrm{cm}$.

For the hexapod configuration (Fig. 21), the weight factor is not as critical as it will at all times be distributed between at least 3 of the limbs and during locomotion the joints are in a position where the weight generated by the configuration does not torque the joints.

\section{CONCLUSION}

Rapid prototyping is a tool that has facilitated the development of complex parts for all kinds of applications, reducing manufacture time and cost. As a manufacturing process it is increasingly being used in different applications and a wide variety of materials.

Modular robotics can benefit significantly from rapid prototyping, since, 3D printing enables rapid manufacture of large quantities of agents to be used in a multi-agent system. In the case of MecaBot-3 modules, experimentation with physical improvements is greatly accelerated. This factor, coupled with advances in materials and prototyping techniques will be paramount in order to provide new features to modular robots in general.

\section{ACKNOWLEDGEMENTS}

This study is a result of the ongoing $\mathrm{NNG}-2376$ research Project, titled "Development of technique for architectural selection and automatic assembly of agents using modular robotics, based on work environment detection", funded by the Vice Deanery for Research of Universidad Militar Nueva Granada.

\section{REFERENCES}

Anonymous, 2016a. [Resilience Unit]. Viajes Tema S.A., Granada, Spain.http://eesaytbailonangeles. blogspot.com.co/2010_10_01_arc hive.html. (In Spanish).

Anonymous, 2016b. [Tips for 3D printing]. Carrer del Poeta Carles Salvador,Valencia, Spain. http://aboratoriodefabricacion.com/servicios/impres ion-3d/consejos-impresion-3d/ (In Spanish).

Krupke, D., F. Wasserfall, N. Hendrich and J. Zhang, 2015. Printable modular robot: An application of rapid prototyping for flexible robot design. Ind. Rob. Intl. J., 42: 149-155.

Matellan, V. and J.M. Canas, 2006. [Modular robotics and locomotion: Robots cube revolutions and multicube]. Ph.D Thesis, King Juan Carlos University, Mostoles, Spain. (In Spanish)

Montana, R.O.G. and E.C.A. Hurtado, 2013. [Design and simulation of a reconfigurable modular robot]. Master Thesis, Nueva Granada Military University, Bogota, Colombia. (In Spanish)

Murata, S. and H. Kurokawa, 2007. Self-reconfigurable robots. IEEE. Rob. Autom. Mag., 14: 71-78.

Yim, M., 1994. Locomotion with a Unit-modular reconfigurable robot. Ph.D Thesis, Stanford University, Stanford, California, USA.

Yim, M., Y. Zhang and D. Duff, 2002. Modular robots. IEEE. Spectrum, 39: 30-34. 